\title{
Light Chain Deposition Disease in Black African, Report of Three Cases from Senegal
}

\author{
Cisse $M^{1 *}$, Fall $S^{2}$, Lemrabott $A T^{1}, K a E F^{1}$, Fall $K^{1}$, Faye $M^{1}$, Niang $A^{1}$ and Diouf $B^{1}$
}

${ }^{1}$ Nephrology Department of Teaching Hospital Aristide Le Dantec, Dakar, Senegal

${ }^{2}$ Internal Medicine Department of Teaching Hospital Aristide Le Dantec, Dakar, Senegal

\begin{abstract}
Background: Although there are numerous publications on multiple myeloma in black people, it seems subSaharan literature particularly Senegalese' one is silent about Light chain deposition disease (LCDD) which is linked to immunoglobulin light chain deposition in glomerulus. The authors report the first three observations of LCDD collected in Nephrology department of Aristide Le Dantec hospital in Dakar, Senegal.

Cases: we report three cases a man and two women of 61,69 and 47 years old respectively, admitted to the Nephrology department of Aristide Le Dantec hospital for rapidly progressive renal failure in one case and a nephrotic syndrome in 2 other cases. The renal biopsy showed a nodular glomerulosclerosis and immunofluorescence microscopy, revealed deposition of IgG light chains suggesting LCDD. On the other hand, the diagnosis of multiple myeloma of IgG kappa type in 2 cases and IgG lambda type in the other one was done. A combination of chemotherapy (Mephalan Prednisone) and hemodialysis was instituted for all 3 cases. The evolution was marked by the appearance of an end stage renal disease in 2 cases and the third one was expired due to an infected bed sore secondary to a pathological fracture of the neck of the femur.
\end{abstract}

Conclusion: Although the LCDD is rare, the prognosis of this syndrome seems to be poor as more than half of the patients die or progress to ESRD within 2 years.

Keywords: Light chain deposition disease; Multiple myeloma; Glomerulosclerosis

Abbreviations: LCDD: Light Chain Deposition Disease; MGUS: Monoclonal Gammopathy of Unknown Significance; MIDD: Monoclonal Immunoglobulin Deposition Disease

\section{Introduction}

Monoclonal Immunoglobulin Deposition Disease (MIDD) is a plasma cell dyscrasia characterized by deposition of monoclonal, amorphous light chains (LCDD) or heavy (HCDD: heavy chain deposition disease) monotypic chain in various organs including the kidney, liver, heart, small intestine, spleen, skin, nervous system, and bone marrow 15, 16, 17, 18, 19 Sites of deposit in the kidney can vary and include the glomeruli, tubular basement membrane, and Bowman capsule.

Approximately $50-60 \%$ of patients with MIDD have associated lymph proliferative disorder, most commonly multiple myeloma. The remaining cases develop MIDD in the setting of progression of monoclonal gammopathy of unknown significance (MGUS) or with no evidence of neoplastic plasma cell proliferation [1]. Renal involvement is constant and often the main clinical and laboratory feature. The latter is typically manifested by massive proteinuria or nephrotic syndrome and sometimes rapidly progressive renal failure with a poor prognosis which is often complicated by the lack of a standard treatment; in more than half of the cases the renal involvement progresses toward endstage renal disease (ESRD) within 2 years [2]. The risk of recurrence after renal transplantation is particularly high [3].

To our knowledge, no cases have been reported in Sub-Saharan Africa. In this paper we describe the clinical, biological, histological, prognostic and therapeutic features of Light chain deposition disease in African blacks by three observations collected in the Nephrology department of Hospital Aristide Le Dantec in Dakar.

\section{Case Presentations}

\section{Case 1}

A 69 years old woman with a past medical history of height blood pressure treating for 12 years was admitted to nephrology department for the management of renal disorders. Clinical examination revealed a glomerular nephropathy syndrome with renal type pitting edema, hypertension, and a $4^{+}$proteinuria yielded by urine dipstick. This glomerular nephropathy syndrome was associated with generalized musculoskeletal pain worsening on movement, markedly in spinal vertebrates and shoulders with weight loss and general weakness.

Laboratory analysis showed a rapidly progressive renal failure with increases in plasma creatinine $(48 \mathrm{~m} / \mathrm{Lg}, 100 \mathrm{mg} / \mathrm{L}, 121 \mathrm{mg} / \mathrm{l})$ and BUN $(0.58 \mathrm{~g} / \mathrm{L}, 1,20 \mathrm{~g} / \mathrm{L}, 1.82 \mathrm{~g} / \mathrm{l})$ in less than a month, the renal failure associated with hyperproteinemia of $81 \mathrm{~g} / \mathrm{l}$, a serum albumin of $39.53 \mathrm{~g}$ $/ 1$, elevated $\alpha 2$ and $\beta 2$ globulin $(11.83 \mathrm{~g} / \mathrm{l}$ and $7.78 \mathrm{~g} / \mathrm{l})$, hyperuricemia of $86 \mathrm{mg} / \mathrm{l}$, hypercalcemia of $107 \mathrm{mg} / \mathrm{l}$ and a normocytic normochromic anemia of chronic disease type with a $\mathrm{Hb}$ of $9.1 \mathrm{~g} / \mathrm{d}$. The bone marrow examination showed $19 \%$ plasma cells with very dysmorphic plasma cells suggestive of multiple myeloma. The $\beta 2$-microglobulin was $10 \mathrm{mg} / \mathrm{l}$ were found in the 24 hour urine collection showed a proteinuria of 3 $\mathrm{g} / \mathrm{l} / 24$ hematuria and pyuria. Urinary protein electrophoresis revealed the presence of $\lambda$ type Bence Jones proteins with a positive free light

*Corresponding author: Mouhamadou Moustapha Cisse, Nephrology Department of Teaching Hospital Aristide Le Dantec, Dakar, Senegal, Tel: 002217757388 09; E-mail:mhmcisse@yahoo.fr

Received October 23, 2013; Accepted November 25, 2013; Published November 29, 2013

Citation: Cisse MM, Fall S, Lemrabott AT, Ka EF, Fall K, et al. (2013) Light Chain Deposition Disease in Black African, Report of Three Cases from Senegal. J Nephrol Therapeutic S1: 003 doi:10.4172/2161-0959.S1-003

Copyright: $\odot 2013$ Cisse MM, et al. This is an open-access article distributed under the terms of the Creative Commons Attribution License, which permits unrestricted use, distribution, and reproduction in any medium, provided the original author and source are credited. 
chain for 17,460 mg/l. abdominal ultrasound and chest radiograph were normal. Radiographs of the skeleton (cervical, lumbar, shoulder, pelvis, and skull) revealed a lumbar osteoarthritis. Retinoscopy demonstrated stage II Kirkendall Hypertensive retinopathy.

Histopathologic examination of renal aspiration biopsy samples showed a myeloma tubulopathy associated with LCDD (Figure 1). Further examination showed congestive heart failure and lower limbs sensory deficit in socks pattern. Thus the diagnosis of a LCDD with renal, cardiac and neurological involvement in the setting of a multiple myeloma was done.

The patient underwent a course of chemotherapy consisted of four days of melphalan $0.20 \mathrm{mg} / \mathrm{kg}$ and prednisone $1 \mathrm{mg} / \mathrm{kg}$, repeated every 28 days. The evolution was marked by the onset of anuria, which brought the patient for hemodialysis. The patient was expired 10 days after finishing the first course of chemotherapy due to the infectious complications following a pathologic fracture of the neck of femur.

\section{Case 2}

A 61 years old patient with no significant past medical history, referred to nephrology department for a massive proteinuria in $9 \mathrm{~g} / \mathrm{l} / 24$ h.

The clinical examination revealed a glomerular nephropathy syndrome with pitting edema, a systolic-diastolic hypertension of $180 / 100 \mathrm{mmHg}$ and no extra renal signs. Urine analysis with dipstick unfolded a proteinuria without hematuria.

Laboratory analysis showed impure proteinuria $9.98 \mathrm{~g} / \mathrm{l} / 24 \mathrm{~h}$, hypoproteinemia of $47.1 \mathrm{~g} / \mathrm{l}$ hypoalbuminemia of $21.9 \mathrm{~g} / \mathrm{l}$, a renal failure with a GFR of $30 \mathrm{ml} / \mathrm{min}$ MDRD) and a decrease in al and $\gamma$-globulins without monoclonal peak in serum protein electrophoresis. $\kappa$ type Bence Jones Proteinuria was noted. The bone marrow showed a slight plasma cells ratio of $11 \%$ without cell dysmorphia. Free $\kappa$ light chains were $470 \mathrm{mg} / \mathrm{l}$ with a $\mathrm{k} / \lambda$ ratio of 10.7 . Abdominal ultrasound was normal.

The histological examination suggested a diagnosis of LCDD with nodular glomerulosclerosis and deposits of $\kappa$ light chains (Figure 2).

The patient was put under nephroprotective and symptomatic treatment (Valsartan, Atenolol, Atorvastatin, and Furosemide and low sodium diet) associated with chemotherapy with melphalan $0.20 \mathrm{mg} /$ $\mathrm{kg}$ and prednisone $1 \mathrm{mg} / \mathrm{kg}$ for 4 days every 28 days. The evolution was marked by the onset of ESRD with a GFR less than $9 \mathrm{ml} / \mathrm{min}$ by MDRD after a decline of 8 months requiring the chronic dialysis.

\section{Case 3}

A 47 years old woman was admitted for anuria. Past medical history disclosed a height blood pressure discovered five months before admission. Physical examination showed a glomerular nephropathy syndrome.

Laboratory data revealed a nephrotic syndrome with a proteinuria of $3 \mathrm{~g} / 24$-hour, hypoproteinemia of $54 \mathrm{~g} / \mathrm{l}$, hypoalbuminemia 23, 71 $\mathrm{g}$, a GFR of (Rapidly progressive acute renal failure)), Normochromic normocytic anemia with $\mathrm{Hb}$ of $6 \mathrm{~g} / \mathrm{dl}$ and a hypercalcemia of 125,44 $\mathrm{mg} / \mathrm{l}$. There was also the presence of $\beta 2$-microglobulin to $36.22 \mathrm{mg} / \mathrm{l}$ and kappa type IgG. Bone marrow plasma cell ratio was $36 \%$ with very dysmorphic plasma cells.

Renal biopsy histological examination displayed a lobular and membranous glomerulonephritis associated with multiple myeloma and LCDD tubulopathy.
In addition to chemotherapy and nephroprotective treatment the patient underwent hemodialysis for 10 sessions. The evolution was marked by a resumption of diuresis without recovery of renal function. The outcome was unfavorable with development of end stage renal disease requiring chronic dialysis.

\section{Discussion}

MIDD is a relatively rare, found only in $5 \%$ of patients with multiple myeloma at autopsy [4-6]. In a Sevillian series, Carmen reported 6 cases of MIDD over a period of six years [7]. LIN in New York reported 34 cases during a period of 18-year [6]. Pozzi et al. in Europe describes the largest cohort with 63 cases in 2003 [2]. To our knowledge, we report the first three cases of Randall Syndrome from Senegal in Sub-Saharan Africa.

The mean age of our patients was 59 years [range 47 to 69]. In the largest series, the mean age ranged between 55 and 58 years (Table 1), and the age group 60-69 years was the most representative $[2,6,8,9]$. The MIDD can also occur in younger patients Indeed. Goosens et al. reported a case in a woman of Somalia origin aged 25 years [10], and Pozzi et al. described cases in patients younger than 40 years [2]. A female predominance is noted with two women and one man
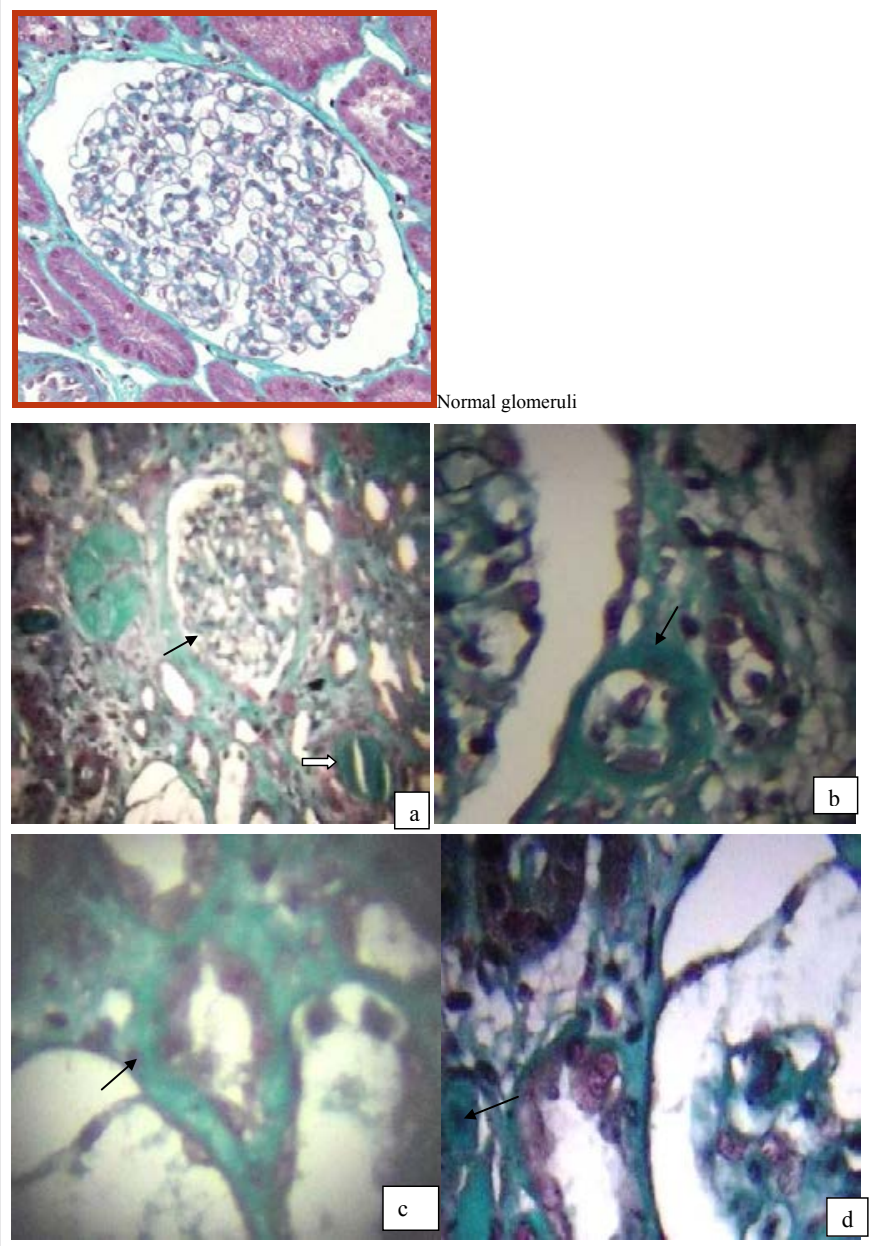

Figure 1: Case 1, Renal biopsy.

(a) Normal glomerulus (black arrow) and cylinder myeloma (white arrow) at low magnification. Masson trichrome x 200 .

(b) and (c) thickening of the tubular glass. Masson trichrome $\times 400$.

(d) Cylinder myeloma. Masson trichrome x 400. 

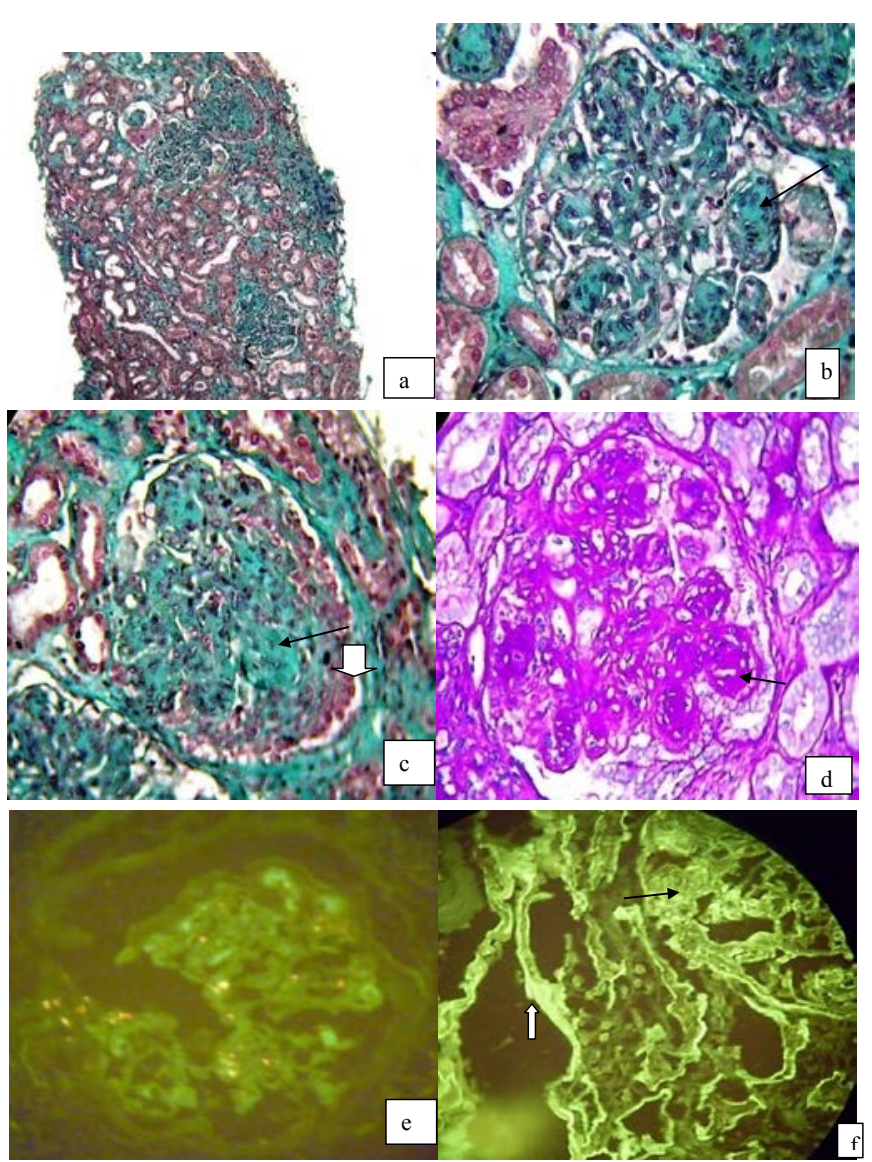

Figure 2: Renal biopsy of patient \# 2. (a), (b), (c), (d), (e), (f)

- Optical microscopy.

(a) Masson trichrome $\times 200$. (b) Nodular glomerulosclerosis (c)

Nodular glomerulosclerosis (black arrow) and increasing fiber-cell (white arrow) Masson trichrome $x$ 400. (d) PAS-positive nodule. NOT x 400 .

- Immunofluorescence:

(e) Absence of $\lambda$ chain deposits (f) deposits $\mathrm{k}$ chains in the glomerulus (black arrow) and tubular basement membranes (white arrow).

\begin{tabular}{|c|c|c|c|}
\hline Country & $\begin{array}{c}\text { reported } \\
\text { Cases }\end{array}$ & $\begin{array}{l}\text { Sex } \\
(H / F)\end{array}$ & Age (age median range) (Year) \\
\hline Italy [8] & 15 & $5 / 10$ & $31-74$ \\
\hline France [9] & 14 & $11 / 3$ & $48-69$ \\
\hline Spain [7] & 6 & $2 / 4$ & $37-74$ \\
\hline Europe [2] & 63 & $40 / 23$ & 58 \\
\hline USA [6] & 34 & $17 / 17$ & $\begin{array}{ll}- & \text { MIDD : } 57,4 \pm 2,12 \\
- & \text { MIDD+ tubulopathy myeloma : } 67,1 \pm 3,92\end{array}$ \\
\hline Senegal & 3 & $1 / 2$ & $\begin{array}{ll}- & \text { LCDD : } 61 \\
- & \text { LCDD + tubulopathy myeloma : } 47 \text { et } 69\end{array}$ \\
\hline
\end{tabular}

Table 1: Monoclonal Immunoglobulin Deposition Disease (MIDD) epidemiology.

in contrast to the series of Pozzi and Necker where there was a male predominance $[2,9]$.

Two of our three patients presented with nephrotic syndrome and one case with rapidly progressive acute renal failure at the time of diagnosis. Indeed, in the series of Pozzi, $52 \%$ of patients had acute or rapidly progressive renal failure. In this series, $84 \%$ of patients had proteinuria $>1 \mathrm{~g} /$ day [2]. In the series of Necker, all patients had renal involvement and 9 of the 14 patients had massive proteinuria [9].

Renal type edema was seen in three patients with milder edema in patients for whom MIDD associated with myeloma tubulopathy (patients No. 1 and 3). This finding is in accordance with the observations of Lin et al. [6] and shows the higher importance of the tubulointerstitial lesions to the less severe glomerular lesions in patients with MIDD associated with myeloma tubulopathy.

Hypertension was observed in all patients. Variable prevalence in the MIDD it must be interpreted in light of past medical history [5].

Extra-renal deposits particularly cardiac were found in our patients. They presented ventricular hypertrophy with diastolic dysfunction in echocardiography, which may reflect a restrictive cardiomyopathy as described in MIDD [11]. Clinically there was no evidence of cardiac involvement. These results are similar to those of Toors et al. who described the same cardiac lesions confirmed at histology in 8 clinically asymptomatic patients with MIDD [12]. No sign and symptom of liver or neurological involvement was found, none the less, maculopapular skin lesions were noted in case N02 which may be the sign of cutaneous deposits as described by some authors $[9,13,14]$. However, histological examination of the lesions was not performed in our patient.

Nodular glomerulosclerosis which is present in all cases MIDD associated with myeloma tubulopathy was seen in two of our patients. The differences in histopathologic aspects of the pure MIDD and those associated with myeloma tubulopathy were also featured in the series of Lin et al. in 2 of 11 patients [6]. Eleven patients showed severe tubulointerstitial lesions with fractured cylinders, edema, interstitial fibrosis and inflammatory infiltrate and the lesions similar to those described in our case N01. In contrast, all 23 patients with pure MIDD had nodular glomerulosclerosis and a variables degree of tubulointerstitial damage, which was dominated by the thickening of the basement membrane [6]. Kappa chain, immunoglobulin G and C3 deposits in tubular basement membrane were seen in two cases and $\lambda$ chain in one case. Electron microscopic examination was not performed for our cases.

Electrophoresis and immunofixation of serum proteins revealed no monoclonal peak in our patients. None the less the Bence Jones proteinuria was detected in all our patients. The serum free light chains were detected in two of our patients. In the series of Pozzi et al., 90\% of patients had a monoclonal component detected in the urine and $76 \%$ in serum [2].

The evolution was marked by a severe impairment of renal function requiring hemodialysis in two patients. Despite the rapid deterioration of renal function, the patient N02 was still alive after 8 months. One patient was expired in less than six weeks after diagnosis because of an infected bed sore following a pathological fracture of the femur neck. These observations are consistent with those described in the literature with survival ranging from 1 month to 10 years [5]. In Pozzi's series, age and initial serum creatinine were the markers of poor renal prognosis [2]. In Lin's one, the presence of myeloma tubulopathy was associated with a poor renal prognosis in addition to the age and initial serum creatinine. In fact 9 of 11 patients with MIDD associated with myeloma tubulopathy were immediately developed ESRD with a renal survival of $10 \%$ within 2 months against $70 \%$ for pure MIDD [6]. The case N01 met all poor prognosis criterias. The patient N02 went toward ESRD 8 months after the diagnosis. That was partly due to the lack of patient adherence to treatment, but largely reflects the severity of MIDD prognosis, even in the absence of Multiple myeloma. In the Lin's series, the two patients with light chain deposition disease (LCDD) associated with MGUS developed ESRD after 2.5 years [4]. Half of patients with LCDD (6/12) and MIDD (11/23) developed ESRD after a mean of 27 
and 22 months, respectively [6]. Also in Pozzi's series, 36 of 63 patients with LCDD went toward ESRD, with an incidence of 23.7/100 patientyears [2].

Various treatments have been tried in MIDD; regardless the presence or absence of multiple myeloma chemotherapy constituted the main treatment in our three patients. The patient N02 underwent a nephroprotective treatment for 6 months, in addition to chemotherapy. In spite these treatments, the renal prognosis remains poor, what confirms the Lin et al. [6] and Pozzi et al. [2] findings.

\section{Conclusion}

It causes a systemic disease in which renal involvement predominates. Renal biopsy plays an essential role in the diagnosis. Nodular glomerulosclerosis and thickening of tubular basement membrane found in half of the cases. Immunohistochemistry can confirm the exclusive presence of monotypic light chain deposition, usually $\kappa$ type and or heavy chains. Myeloma tubulopathy lesions may be present and in this case seems to be associated with a poor prognosis. Despite its rarity, the prognosis of this disease is poor because as than half of the patients die or progress toward ESRD within 2 years.

\section{References}

1. Swerdlow SH, Campo E, Harris NL, Jaffe ES, Pileri SA, et al. (2008) WHO Classification of Tumours of Haematopoietic and Lymphoid Tissues. Lyon, International Agency for Research on Cancer 200-213.

2. Pozzi C, D'Amico M, Fogazzi GB, Curioni S, Ferrario F, et al. (2003) Light chain deposition disease with renal involvement: clinical characteristics and prognostic factors. Am J Kidney Dis 42: 1154-1163.

3. Leung N, Lager DJ, Gertz MA, Wilson K, Kanakiriya S, et al. (2004) Long-term outcome of renal transplantation in light-chain deposition disease. Am J Kidney Dis 43: 147-153.

4. Jimenez-Zepeda VH (2012) Light chain deposition disease: novel biological insights and treatment advances. Int J Lab Hematol 34: 347-355.

5. Ronco P, Plaisier E, Mougenot B, Aucouturier P (2006) Immunoglobulin light (heavy)-chain deposition disease: from molecular medicine to pathophysiologydriven therapy. Clin J Am Soc Nephrol 1: 1342-1350.

6. Lin J, Markowitz GS, Valeri AM, Kambham N, Sherman WH, et al. (2001) Rena monoclonal immunoglobulin deposition disease: the disease spectrum. J Am Soc Nephrol 12: 1482-1492.

7. Carmen MH, Marta SP, Margarita DP, Jorne FA, Rocio C (2008) Light chain deposition disease: Experience in our environment. Nefrologia 28: 539-542.

8. Confalonieri R, Barbiano di Belgiojoso G, Banfi G, Ferrario F, Bertani T, et al (1988) Light chain nephropathy: histological and clinical aspects in 15 cases. Nephrol Dial Transplant 3: 150-156.

9. Ganeval D, Noël LH, Preud'homme JL, Droz D, Grünfeld JP (1984) Light-chain deposition disease: its relation with AL-type amyloidosis. Kidney Int 26: 1-9.

10. Goosens AN, Weening JJ, Schut NH (1998) A young woman from Somalia with acute renal failure. Nephrol Dial Transplant 13: 1832-1834.

11. Fabbian F, Stabellini N, Sartori S, Tombesi P, Aleotti A, et al. (2007) Light chain deposition disease presenting as paroxysmal atrial fibrillation: a case report. $J$ Med Case Rep 1: 187.

12. Toor AA, Ramdane BA, Joseph J, Thomas M, O'Hara C, et al. (2006) Cardiac nonamyloidotic immunoglobulin deposition disease. Mod Pathol 19: 233-237.

13. Randall RE, Williamson WC Jr, Mullinax F, Tung MY, Still WJ (1976) Manifestations of systemic light chain deposition. Am J Med 60: 293-299.

14. Safa G, Dhib M, Soubrane JC, Thomine E, Delpech A, et al. (1996) [Light and heavy chain deposition disease with cutaneous and renal manifestations]. Ann Dermatol Venereol 123: 490-492. 